\title{
A retrospective study concerning specific therapy and evolution of invasive fungal infections diagnosed in the National Institute for Infectious Diseases "Prof. Dr. Matei Balş"
}

\author{
Ramona Zamfir ${ }^{*}$, Olga Dorobățt ${ }^{1}$, Daniela Tălăpan ${ }^{1}$, Roxana Dumitriu ${ }^{1,2}$, Alexandru Rafila ${ }^{1,2}$, Elisabeta Benea ${ }^{1,2}$ \\ From The 10th Edition of the Scientific Days of the National Institute for Infectious Diseases "Prof Dr Matei \\ Bals" \\ Bucharest, Romania. 15-17 October 2014
}

\section{Background}

Invasive fungal infections still have high morbidity and mortality rates, especially in immunocompromised patients, given the lack of specific symptomatology and of fast and early diagnosis methods.

\section{Methods}

We present a retrospective study performed between January 2011 - June 2014 in the National Institute of Infectious Diseases "Prof. Dr. Matei Balş", including patients with invasive fungal infections and complete clinical and biological data.

\section{Results}

18 patients met the inclusion criteria for the studied period, representing $40 \%$ of the patients with potentially invasive fungal infections. The majority of patients were male, and the average age was 44 years. Only 2 of the patients apparently were not immunocompromised, the other 16 presenting HIV infection or fungal infections risk factors. The fungal species identified were Cryptococcus neoformans in 8 cases (40\%) and Candida spp in 12 cases (60\%), out of which $75 \%$ consisted of non-albicans species. The invasive fungi were isolated from blood cultures in 9 cases (52.4\%), cerebrospinal fluid in 8 cases (38.1\%), tips of central venous catheters in one case and from other pathological products in one case. The average period from admission to identification was 9.6 days. The antifungal

\footnotetext{
* Correspondence: ramzamfir@gmail.com

'National Institute for Infectious Diseases "Prof. Dr. Matei Balş", Bucharest, Romania

Full list of author information is available at the end of the article
}

susceptibility test indicated that 16 out of the 20 cases $(80 \%)$ were sensitive to fluconazole and only $20 \%$ were dose-dependent sensitivity types. The patients were treated mainly using monotherapy - 1 antifungal in 10 cases (55\%). Fluconazole was the most used agent, in 14 cases (77.8\%) followed by voriconazole in 7 cases and posaconazole in 4 cases. The average treatment duration was 37.7 days, the shortest being one day, and the longest 120 days. 6 out of 18 patients (33.3\%) deceased: one patient presenting severe bacterial infection treated with prolonged antibiotherapy and 5 presenting C. neoformans meningitis associated with HIV infection.

\section{Conclusion}

Although proper treatment was administered, the mortality in invasive fungal infections remains high, given the fact that they generally appear in already marred or severely immunosuppressed patients.

\section{Authors' details \\ 1National Institute for Infectious Diseases "Prof. Dr. Matei Balş", Bucharest, Romania. ${ }^{2}$ Carol Davila University of Medicine and Pharmacy, Bucharest, Romania.}

Published: 15 October 2014

doi:10.1186/1471-2334-14-S7-P4

Cite this article as: Zamfir et al:: A retrospective study concerning specific therapy and evolution of invasive fungal infections diagnosed in the National Institute for Infectious Diseases "Prof. Dr. Matei Balş". BMC Infectious Diseases 2014 14(Suppl 7):P4. 